\title{
Technique of implantation of extrapupillary iris lens: 8 years of clinical experience
}

\author{
M. M. KRASNOV \\ From the State Institute of Eye Diseases, Moscow
}

SUMmARY A new type of intraocular lens has been devised. The lens is iris-supported but, in contrast to conventional iris-clip lenses, is fixed at the iris periphery, leaving the pupillary region free. The lens may be implanted either anterior or posterior to the iris plane. Implantation into the posterior chamber is particularly beneficial when combined with an extracapsular extraction. Eight years' experience (189 operations) underlines a number of important advantages of the extrapupillary iris lens. The pupil is not distorted, so that its form and function remain normal; the danger of dislocation is avoided; and the lens may be employed in the presence of an iris coloboma. The supporting prongs of the lens are made of gold and platinum alloys, which add considerably to its tissue tolerance. So far no complication has arisen necessitating removal of such a lens. This new type of intraocular lens offers a combination of efficacy, safety, and technical simplicity.

The 'extrapupillary iris lens' was first mooted in 1968. Although it is an iris-supported lens, the points of attachment are placed at the periphery of the iris away from the pupillary region. As compared to the conventional iris-clip lenses this attachment has major advantages (US Patent No. 3922728) (Krasnov, 1975). The main advantages of the extrapupillary iris lens over the conventional irisclip lenses are as follows:

(1) The form and function of the pupil remain normal. (2) Dislocation of the extrapupillary lens is rare. The incidence of dislocation of the iris clip lens is 5 to $10 \%$ (Fyodorov and Feldman, 1973; Zakharova and Fyodorov, 1973; Nordlohne, 1975). (3) The vitreous face is not damaged by the extrapupillary iris lens because no large supporting loop is introduced behind the iris, so that the chance of vitreous loss is minimised. (4) Mydriatics may be used postoperatively to suppress inflammation, whereas mydriatics may lead to complications with iris-clip lenses (Fyodorov, 1973). (5) The extrapupillary iris lens may be employed in the presence of an iris coloboma. (6) Absence of contact of the extrapupillary iris lens with the anterior chamber angle avoids structural and functional damage to this area. (7) The lens design is simple and made of biologically inert materials (highly purified methyl methacrylate with platinum or gold alloys). (8) The

Address for reprints: Professor M. M. Krasnov, State Institute of Eye Diseases, 5 Pogodinskaja Street, Moscow 119453, USSR surgical procedure is technically uncomplicated. All manipulations are limited to the confines of the anterior chamber.

The general trend of development in the field of intraocular lenses is towards extrapupillary fixation, as is exemplified in the suturing of iris-clip lenses (Worst, 1971) to the periphery of the iris. Ten years ago clinical trials of several types of extrapupillary iris lenses were instituted. The original model had three supporting prongs inserted into punctures at the iris periphery. Two fixation points, however, were found to be ample. A whole family of extrapupillary lenses was tested clinically (Fig. 1), and eventually two were found to be the most practical (Fig. 2).

The first (Fig. 2A) has two bayonet-type fixation prongs. The prongs (made of platinum or gold alloys) have pointed ends introduced into corresponding holes in the iris periphery. The correct placing of these holes constitutes a delicate step in surgical technique. If the holes are placed too far apart the fixation of the lens may not be firm enough, especially in mydriasis. If they are placed too close together the pupil may become elongated (which is not dangerous, but cosmetically unsatisfactory). In the second type (Fig. $2 B$ ) one of the supporting prongs is made in the form of a loop to be sutured to the iris (usually above near the incision); the opposite (distal) prong is the same as in type $A$, and is introduced into a dehiscence in the iris. One of the prongs is thus rigidly attached; the other is 


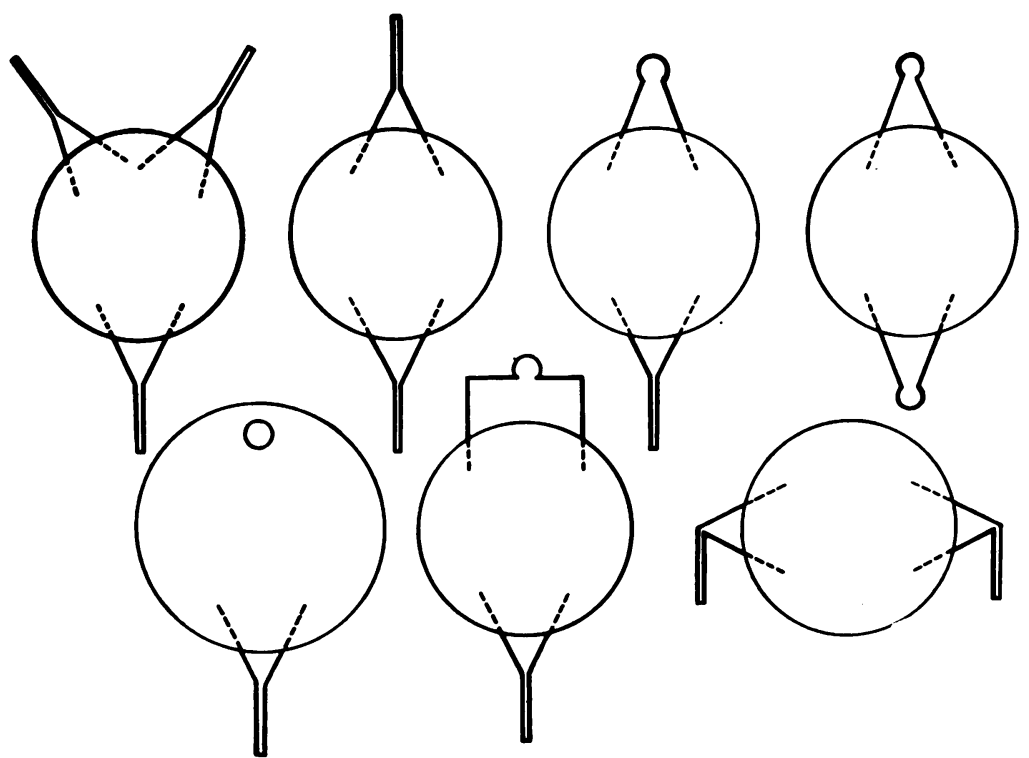

Fig. 1 A family of extrapupillary iris lenses with different types of supporting prongs

referred to as the 'sliding' prong. The technique of implantation of the $B$ type extrapupillary lens requires more time because of the iris suture, but on the whole the procedure is quite safe in routine practice. The $B$ type lens lessens the risk of dislocation. An iris suture, of course, may be inserted through holes in the body of the lens itself as in the Worst 'Medallion' lens. Movement of the iris musculature influences the lens less if the attachment to the iris is well away from the pupil border. In order to make the fixation point as peripheral as possible it is preferable to pass a suture through an 'intermediate' loop-like prong which extends to the iris root. If such a suture to the iris periphery has an attachment to the body of the lens, then the optical portion is of necessity unduly large and heavy.

A small iridotomy is usually done at the site of suturing to sever the iris muscles from the fixation point. The type $B$ is especially advantageous in cases with an iris coloboma or traumatic mydriasis and in situations wherein special safety requirements are necessary, for instance, for sportsmen, drivers, or pilots. It is possible to suture the loop-like supporting prongs to the iris, but the complexities of this technique do not seem to justify its routine use. As we pointed out elsewhere (Krasnov and Orlova, 1969) an extrapupillary iris lens may also be placed in the posterior chamber.

Fig. 2 Bayonet-type of extrapupillary iris lens (A) and lens with a loop-like prong for an iris suture (B)

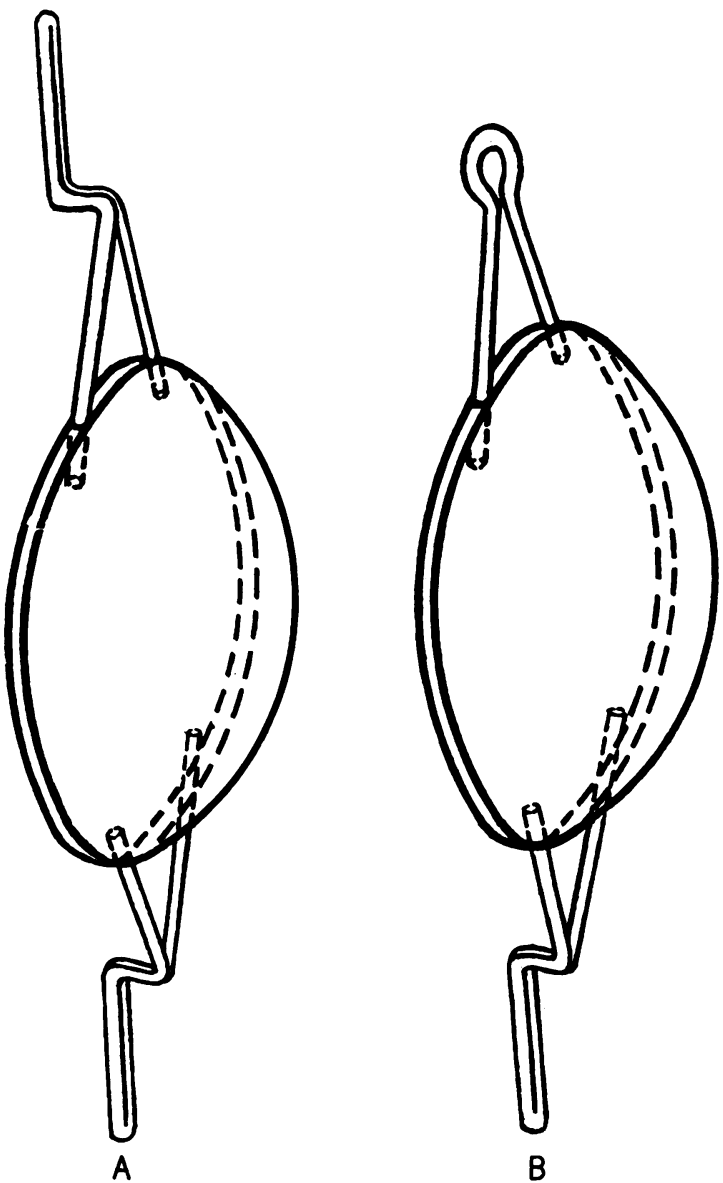




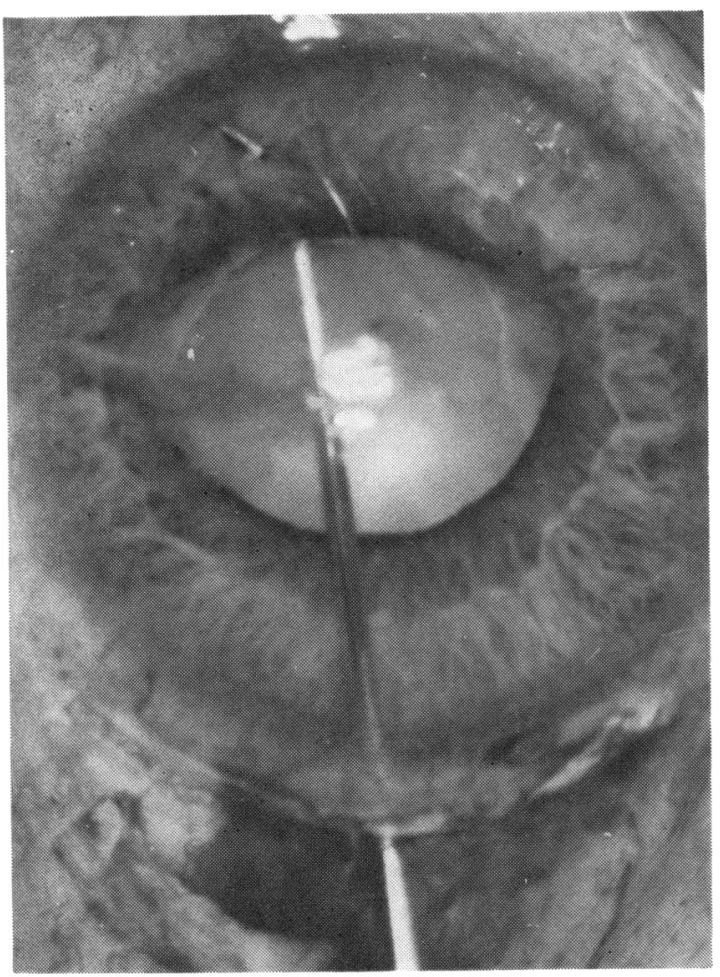

Fig. 3 Making of distal iridopuncture by a Sato knife

A total of 189 extrapupillary iris lens implantations have been inserted and followed up during the last 8 years. In no case has a lens had to be removed because of complications. There were no cases of bullous keratopathy. During the first years of experience in 9 cases the upper supporting prong was displaced soon after insertion owing to poor surgical technique in placing the iridopunctures. As soon as the cause was recognised, this surgical error was avoided.

The primary aim of this paper is to describe the essential elements of the surgical technique for the implantation of an extrapupillary iris lens. Standard surgical techniques such as adequate hypotony of the eye before operation, proper anaesthesia, and perfect suturing of the incision are taken for granted, and only specific points are described. All types of extrapupillary lenses can be implanted simultaneously with cataract extraction or as a separate procedure later.

\section{Operative technique}

Iridopunctures. A pinpoint hole should be made at the periphery of the iris before the anterior chamber is emptied. The anterior chamber is entered by a
Sato-knife (or a similar type of needle/knife), usually at the upper limbus. The instrument then is slowly carried across the anterior chamber to the pupil, and from here on it passes under the iris to the point of planned iridopuncture (Fig. 3). At this point the shaft is rotated through $90^{\circ}$ and the blade of the instrument cuts through the iris from behind (towards the cornea). The position of the iridopuncture must be approximately half-way between the pupillary margin and the limbus of the same meridian. It is important to enlarge the opening (sometimes by a blunt spatula) to make it wide enough to accept the supporting prong of the lens without unnecessary trauma to the iris. If the anterior chamber is accidentally emptied during the manoeuvre it is more difficult to perform an iridopuncture. The anterior chamber should then be refilled to its full depth (if necessary, some temporary sutures can be inserted to prevent the escape of fluid) and the procedure repeated in exactly the same way. A good iridopuncture may be produced by laser (Krasnov, 1973) preoperatively.

If the $A$ type of extrapupillary iris lens is used (bayonet type of fixation of both supporting prongs), then a second iridopuncture is carried out after the incision into the anterior chamber has been completed. A second (proximal) iridopuncture is easy to perform because it is situated quite close to the incision.

The iris is grasped by fine forceps, lifted up slightly, and punctured either by a Sato-knife or pinched by Vannas scissors. If the $B$ type is used, an iris suture is placed at the same point to secure the proximal supporting prong of the lens later (see below).

A Supramid or nylon thread can readily be used, but sutures of 20 to $25 \mu \mathrm{m}$ wire (gold and platinum alloys especially) are superior in our hands. A basal iridectomy or an iridotomy is often performed at this meridian, and then the suture is conveniently passed through the distal (central) edge of the iris coloboma.

As already indicated, when a cataract extraction is completed and the pupil made miotic, all the manipulations with an extrapupillary iris lens are confined to the anterior chamber entirely. This is a factor of great safety, as no supporting loops are pushed behind the iris into the vitreous chamber as is the case with the iris-clip lenses.

Manoeuvring of the lens into the anterior chamber. Originally, the lens was slid into the anterior chamber with its 'bayonet' prong pointing to the site of the iridopuncture (the pupil being avoided so as not to injure the vitreous face). The main technical inconvenience of this approach was the tendency of the pointed end of the prong to become entangled in the iris tissue. It proved both easier and safer to 
introduce the lens with the supporting prongs in the plane of the corneoscleral incision and pointing to 3 and 9 o'clock. The lens is then rotated in the anterior chamber, so that the bayonet prong approaches the iridopuncture laterally. In this way the tendency for the prong to be entangled by iris folds is lessened.

Manipulations with the lens inside the anterior chamber. 'Hydraulic separation' of the structures of the anterior segment of the eye. When an intraocular lens has been manoeuvred into the anterior chamber damage to the cornea, the iris, and the vitreous body must be avoided. If the eye is hypotonic the lens slides in easily, and the supporting prongs go into the preplaced holes without difficulty. If the iris is forward towards the cornea, there is a tendency for iris tissue to obstruct the ends of the supporting prongs. Occasionally difficulties are met with in placing a bayonet prong if the accepting iridopuncture is too small. The Binkhorst forceps then prove helpful.

The best method of coping with such problems is to restore the normal depth of the anterior chamber with reposition of all its structures. It is possible, for instance, to put a lens into the anterior chamber, seal the wound, fill the anterior chamber with saline, and then proceed with the positioning of the lens using thin spatulae. Alternatively, 'hydraulic restoration of the anterior chamber' is most efficient. The incision is not sutured, but adequate separation of the iris and cornea is achieved by a constant inflow of fluid. The assistant fills the anterior

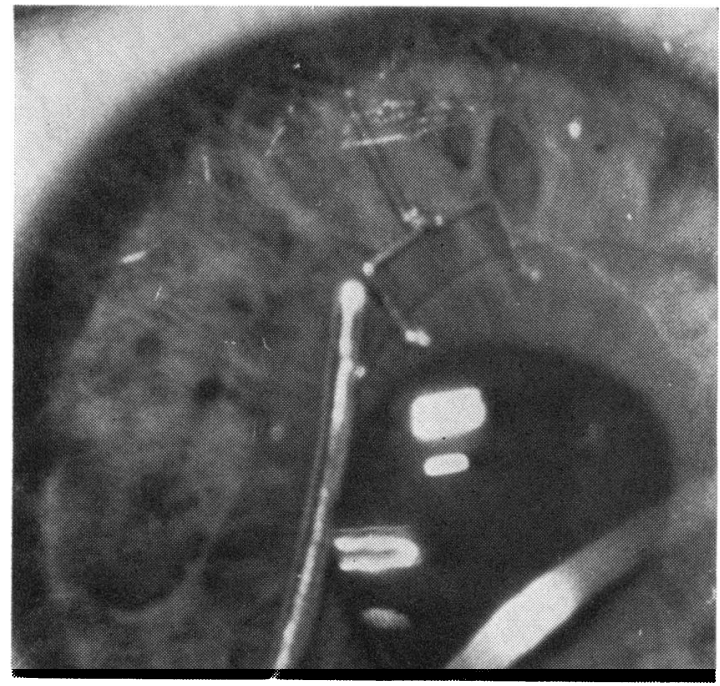

Fig. 4 Hydraulic separation of the cornea and iris as distal supporting prong is being manoeuvred into the iris puncture

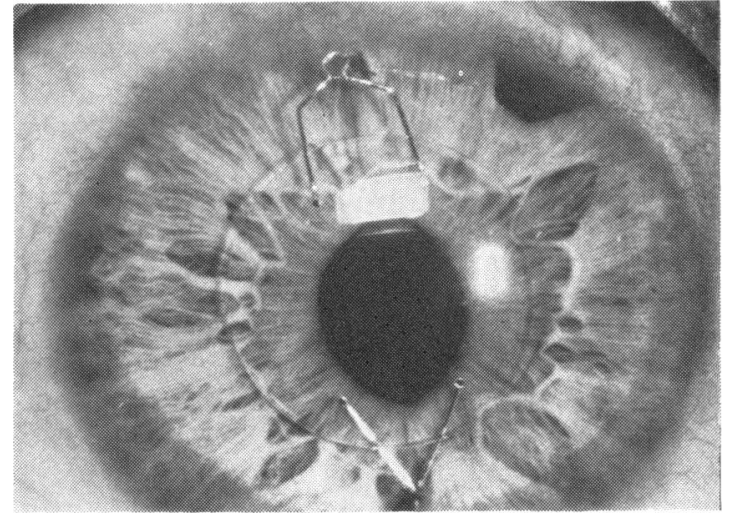

Fig. 5 End result of implantation of extrapupillary iris lens (B) with sutured upper supporting prong

chamber while the surgeon concentrates his attention on manipulating the probe (Fig. 4). After the bayonet prong is secured in the iris hole, the lens must be properly centred in the anterior chamber.

Fixation of the proximal supporting prong (versions A and B). Version A (two bayonet prongs). When the distal prong is secured, the upper part of the iris is pulled out of the wound slightly by iris forceps. The proximal iridopuncture can thus be moved to the end of the proximal prong, which finally slides into it. After that the iris is gently reposited.

Version B (one bayonet prong, one loop for suturing). The iris suture is placed before the intraocular lens is positioned in the anterior chamber. The end of this suture is passed through the loop (but left untied) prior to the manipulations described above. After the distal prong of the lens is secured and the lens itself centred the only step left is to tie the knot. If sutures of gold or platinum alloys are used 1 knot is sufficient. Nylon or Supramid sutures are tied with 2 or even 3 knots. The end of the cut suture must not touch the cornea or the structures of the anterior chamber angle. Suturing of the lens gives an additional element of safety; no dislocation was encountered with this type of fixation (Fig. 5).

Technique of extrapupillary lens implantation into the posterior chamber. The $B$ type of extrapupillary iris lens (one bayonet prong, one loop for suturing) is fairly well suited for implantation into the posterior chamber. As is known, this is especially advantageous with an extracapsular lens extraction. In this particular situation there is no need for iridopunctures.

The lens is gently pushed in through the incision and then through the pupil (with the bayonet prong directed forward into the posterior chamber). A suture should then be passed through the loop-like prong to control the lens position. When the lens is 
completely covered by the iris the bayonet prong perforates the lens capsule at its equator. After the lens is properly centred its proximal (usually, upper) loop-like prong is sutured to the edge of the iris coloboma.

It is commonly considered that the position of the lens in its natural place, especially in combination with an extracapsular extraction of cataract, has the advantages of lesser degrees of aniseikonia and less risk of retinal and vitreous complications.

Final stages of the operation. A watertight closure of the wound is imperative. Special attention should be given to evacuation of all traces of blood from the anterior chamber. Filling the anterior chamber with air may be risky. Pupillary blockage is particularly undesirable in the presence of an intraocular lens (and may among other things predispose to lens dislocation). Air injection should be recommended. After the toilet of the incision and the anterior chamber is completed, the air is replaced by saline or (preferably) by a balanced salt solution. Implantation of the extrapupillary iris lens into both the anterior and posterior chamber of the eye can readily be combined with phakoemulsification (Krasnov et al., 1975).

In conclusion it may be stated that the extra- pupillary iris lens is one of the most practical and safest solutions to the problem of intraocular lens implantation.

\section{References}

Fyodorov, S. N. (1973). Contemporary state of intraocular lenses. In Reconstructive Surgery and Alloplastics in Ophthalmology, pp. 41-47. Moscow Institute of Stomatology: Moscow.

Fyodorov, S. N., and Feldman, B. G. (1973). Complications during a follow-up of patients with intraocular lenses. Vestnik Oftalmologii, 69-71.

Krasnov, M. M. (1973). Laser microsurgery of the eye. Vestnik Oftalmologii, 4-11.

Krasnov, M. M. (1975). Extrapupillary iris-lens. Vestnik Oftalmologii, 42-47.

Krasnov, M. M., Bocharov, V. E., and Dvali, M. L. (1975). Phacoemulsification with implantation of intraocular lens. Vestnik Oftalmologii, 29-32.

Krasnov, M. M., and Orlova, E. M. (1969). Intraocular lens with attachment to the iris outside pupillary region. Vestnik Oftalmologii, 47-51.

Nordlohne, M. A. (1975). The Intraocular Lens. W. Junk: The Hague.

Worst, J. G. F. (1971). Note on fixation of the Binkhorst iris-clip lens. Ophthalmologica, 163, 10-11.

Zakharova, E. J., and Fyodorov, S. N. (1973). Results of 65 implantations of iris-clip lens of a new type. In Reconstructive Surgery and Alloplastics in Ophthalmology, pp. 48-55. Moscow Institute of Stomatology: Moscow. 